\title{
Index Volume I
}

(Numbers followed by asterisk refer to Society Abstracts)

Aagenaes, O. 409*

AbildgaArd, C. F. 39,99

Abortion 207*, 208*, 210*

Abrams, I. 169

Acetazolomide 224*

Aceto, T., Jr. 205*

Acetylcholine 222*

Achlorhydria 306*

Acid-Base measurement $412 *$

Acid-Base metabolism 419*

Acid-Citrate dextrose 333

Acid loading 212*

Acidosis

- general $221 *, 224 *, 277,333$, $415^{*}, 418,452$

- metabolic $212 *, 277,410^{*}, 411^{*}$

- renal tubular 81

ACTH 204*

Actinomycin D 220*

ADAMS, F.H. 364

AdAmson, L.F. 220*

ADdanki, S. 224*

Adenosinetriphosphatase 27, 116, 436

Adenosinetriphosphate 24

Adolescence 203*

Adrenal gland

- general $207 *$

- hyperplasia, congenital 204*, 415*

- medulla 293*

- removal 216*

Adrenaline, excretion 293

Agathopoulos, A. 418*

Akryama, Y. 225*

$\beta$-Alanine $212 *$

Album, M. M. 314*

Albumin test 413*

Alcohol dehydrogenase 165

Aldosterone 207*

Alexander, E. R. 210*

Alford, C. 209*

Alkalosis 204*

Alkalosis, respiratory 354

Allen, D. M. 214*

Allergy 216*

Altay, C. 213*

Alveolar cell 201*

Alveolar gas tensions 201*

Alveolus 131

Amino acids

- general 212*, 220*

- transport $417 *$

- urine 219*, 306*, 425

$\beta$-Aminoisobutyric acid 212*

o-Aminophenol 204*

Ammonium chloride 212*, 452

Amniotic fluid 215*

Amylo-1,6-glucosidase 413*

AnAST, G. S. 203, 220*

Andersen, D.W. 266

Anderson, G.J. 386

Anderson, J.A. 372

Androgen 203*, 214*
Anemia

- aplastic 214*

- general 208*, 214*, 333, 479

- iron deficiency $214 *$

- megaloblastic 306*

- nutritional 169

Anencephaly $308 \%$

Angiotensin 202*

Antibody 50, 178, 209*, 215*, $216 *, 217 *, 319$

Antifibrinogen serum 211*

Antigen 216*

Antihemophilic factor 213*

Antilymphocyte serum 218*

Antistreptolysin $0211^{*}$

Apnea 222*

Apoceruloplasmin 219*

AREY, J. B. 317*

Arginine 205*, 299*, 414*

ARIAS, Y. M. 204*

ARNEIL, G. 414*

Ascites 215*

Askin, P. 50

Aspartate transcarbamylase $414^{*}$

Asphyxia 221*, 226*

Asthma 201*

Atelectasis 418*

Athyreosis 271

Atrioventricular block 202*

Auditory nerve 223*

Autoantibody $418 *$

Autofluorescence 206*

Autosomal recessive inheritance 213*

BACK, N. $317 *$

Bacilluria 303*

Bacterial flora, inhibition 310 *

BADOUAL, J. 413*

BAEHNER, R. L. 306*

BAILEY, J.D. $300^{*}$

BaIN, H.W. 300*

Balassanian, N. 309*

Bargman, G.J. 223*

BARR, R. G. 202*

BARRETT, C.T. 223*

Bartonellosis 295*

Basal metabolic rate 185

Base excess 333, 412*

BASHORE, R. 212*

BAUER, R. 200*

BAUM, R. S. 288*

BAZELON, M. 207*

BEARG, S. 185

BEHRMAN, R.E. 354

Belay, M. 411*

Belloni, C. 419*

BENKE, P.J. 436

Berendes, H.W. 214*

Bicarbonate 81

Bilirubin 204*, 213*, 215*, 409*, 410*

Blanc, W. A. 218*, 222*

Blankenship, W.J. 311 *
Blood groups 5

Blood pressure 122, 411*

Blood volume 223*, 411*

BoDA, D. 411*

Body surface area 185

Body weight 185

BoEsman, M. 216*

BogGs, T.R. 219*

Borchrs, H. 81, 298*, 452

BoIGNe, J.-M. 271

Bone

- abnormality 304*

- collagen 219*

- cortical 479

- general 266

- marrow 218*

Bongrovanni, A. M. 299*, 314*

BOONYAPRAKOB, U. 200*

Borrone, G. $416 *$, 418*

Boumasin, J.D. 213*

BoUlange, M. 415*

Brain 218*, 266*

BRAUDO, L. 212*

BRICKMAN, A. 209*

Brigand, E. P. 215*

BRODEHL, J. 409*

Bronchial secretions 201*

Brown, C.D. 302*

Brown, D. M. 395

Brubaker, C. 116

BRUHL, H. 372

Brunell, P.A. 209*

BRYSON, M.F. 308*

ButLer, B.V. 225*

Cadotte, M. 222*

CAaHILL, F.D. 224*

Calcification 219*

Calcium 203*, 414*

Candidiasis, mucocutaneous 217*

Canales, L. 216*

Canent, R.V., Jr. 202*

CANN, H. M. 206*

CApp, M.P. 202*

Carbamyl aspartate $414^{*}$

Carbohydrate metabolism 116

Cardiac catheterization 122

Cardiac function 202*

Cardiac output 122, 287*

Cardioversion 301*

Garpenter, D. G. 5

Carter, C.H. 5

Cataract 14, 217*

Cave, P. 222*

Gelander, O. 411*

Gentral nervous system 206*, 209*, 224*

Ceramide 416*

Gerebral palsy 5

Cerebro-hepato-renal syndrome 206*

Cerebrospinal fluid 224*

Cervical infection 210* 
Ceruloplasmin 219*, 224*

Chandor, S. B. 217*

Gharache, P. 219*

CHENG, J. 216*

Ghentow, S. 211*

GHernick, V. 201*

GHerRy, R. B. 223*

GHIDA, N. 364

ChILgRen, R.A. 217*

Chimerism 317*

Gholine 364

Chondroitin sulfate 203*, 418*

Choreoathetosis 5

Ghristiansen, P.M. 418*

Cimristiansen, R. O. 292*

Chromosomes 104, 207*, 208*, 461

Clankson, P. M. 320*

Cleft lip 206*

Cleft palate $206 * 317 *$

Coagulation $39,99,211 *, 213 *, 221 *$

Cochlea 223*

Coren, F. 301*

Cohen, M. M. 104

Colitis, ulcerative 418*

Collagen 266

Collipp, P.J. 116

Colon 418*

Colostrum 217*

Communication, doctor-patient 297*

Conjunctivitis 209*

Convulsions 104, 220*

Cook, C. D. 290*

Cooke, R. 418*

COOPER, L.Z. 217*

Copper 219*, 224*

Corpuscle, colostral 217*

Corrigan, J.J., Jr. 39, 99

Cortical necrosis, renal 99

Corticosteroid 204*, 215*, 216*, $317 *, 323 *$

Cortisone 99

Cortner, J.A. 205*

Coscia, A. 323*

Cough 201*

Courtecuisse, V. 414*

CowIE, V. 207*

Graniopharyngioma 300*

GRAWFORD, J. D. 291*

Cress, H. 173

GROPP, G.J. 201*

CROWELL, D. 225*

CuIllier, J. 415*

Cuisinier-Greizes, P. 415*

Cycloleucine 220*

Cyclophosphamide 211*

Cystic fibrosis of the pancreas 24 $173,314 *, 436$

Cystinosis $315^{*}$

Cytomegalic inclusion disease $210 *, 217 *$

Cytosine diphosphate 247

DaILy, W.J. 222*

DAMESHEK, W. $214 *$

Davidson, R. G. 205*

Davis, M.S. 297*

Dawson's inclusion body encephalitis $315^{*}$
Deafness, nerve 306*

Dehydroepiandrosterone sulfate $203 *$

Della Gella, G. 416*, 418*

Denning, C.R. 201*

Deoxyribonucleic acid 217*

Dermatoglyphics 461

Destine, M. L. 317*

Diabetes

- general 110

- mellitus $213 *, 312 *, 470$

- pseudophlorizin 304

Diarrhea $412 *, 417 *$

2,3-Diphosphoglycerate 410*

DNA 461

Dodge, W.F. 211*

Doeden, D. 372

Donneli, G.N. 116

Downes, J.J. 219*

Down's syndrome 207*, 302*

Ducharme, J.R. 204*

Duchenne dystrophy $415 *$

Duodenum 412*

Durand, P. 416*. 418*

Dushane, J.W. 320*

Dwarfism 300*

Dysproteinemia 211*

Eberlein, W.R. 203*, 299*

EBRey, P. 411*

ECK, E. 411*

E. Coli 413*

Eczema 210*

EdelmanN, C. M., Jr. 81, 298*, 452

EDWARDS, S. B. 202*

Eidelman, A. 290*

Electrical countershock $301 *$

Electroencephalogram 225*, 226*, 416*

ELLIS, E.F. 178

Embryo, human 216*

Emmanouilides, G. 200*

Encephalitis 209*, 315*

Encephalomyocarditis virus 319*

Endocardial fibroelastosis $202 *$

Endoplasmic reticulum 219*

Endotoxin 39, 99

ENGEL, K. 412*

ENGEL, R. 225*

Enzyme induction 221*

Epicanthic folds 206*

Epinephrine 202*, 216*, 312*

Epiphyseal maturation 470

Erythema multiforme 210*

Erythrocyte 14, 202*, 215*, $220 *, 410^{*}$

Erythropoiesis 214*

EshaghPOUR, E. 222*

Ethanol 165, 413*

Euglobulin 173

Evoked potentials 225*, 416*

Exanthem 210*

Exchange transfusion 223*, 411*, $419 *$

Fabry's syndrome $416^{*}$

Factor V 99

Factor VIII 99
Factor XIII 213*

FARIDY, E.E. 201*

FARQUHAR, J.W. 110

Fat absorption 220*

Fat, brown $411 *$

Fatty acid, nonesterified $219 *$ $220 *, 411 *$

FAULKNER, S. 415*

FAWCETT, J. 219*

Feinstern, D. $217 *$

Fellers, F.X. 304*

Fernbach, D. J. 169

Ferreiro, M. 215*

$\alpha$-Fetoprotein $216^{*}$

Fetus 165, 208*, 287*, 293*, $308 *, 354,364,443$

Fibrin 211*

Fibrinogen 99, 221*

Fibroblast 203*, 208*

FidAlgo, B.V. 295*

FiLeR, L.J., Jr. 266

FINE, M. H. 214*

Frsh, A.J. 210*

FishMAN, A. P. 201*

Flare 216*

Fletcher, G. 222*

Fojaco, R. 222*

FolTz, E. 224*

FOLWELL, J.A. 203*, 220*

Fomon, S.J. 266

FONG, S. 215*

Fraser, D. 425

FREEMAN, J. M. 315*

FRENGH, J.H. 206*

FRENGK, N. 411 *

FRÉZAL, J. 27

Friedman, S. B. 319*

Frits-Hansen, B. 418*

Frohman, L.A. 205*

Fructose $223^{*}$

Fructose-1,6-diphosphatase 116

Fructose-6-phosphate 202*

FRYE, R. L. 320*

Fulginiti, V.A. 50

Gagliardino, J.J. 300*

Galactitol 14

Galactocerebroside sulfokinase 223*

Galactokinase 14

Galactose 14, 220*

Galactosemia 409*

Galactose-1-phosphate 220*

Galactose-1-phosphate uridyltransferase 14, 220*, 409*

Galan, E. M. 304*

Galofre, A. 414*

Gamma-globulin 110, 295*

Garcia-Castro, J. M. 206*

GARDNER, L. I. 223*

GARTNER, L. M. 204*

Gastric secretion 418*

Gellissen, K. 409*

Gene 205*, 206*

Genetics 104, 213*, 306* 314*

GERALD, P.S. 207*

GidAY, Z. 386

GILCHRIST, G.S. 116

GibBs, G.E. 24 
Giles, J. P. 294*

Githens, J.H. 218*

GrteIN, D. 218*

Gitzelmann, R. 14, 409*

GLASGOW, L.A. 319*

Glomerular filtration rate 185

Glomerulonephritis 210*, 211*, $212 *$

Glucagon 220*

GLUCK, J. 204*

GLUCK, L. 237, 247, 290*

Gluconeogenesis 212*, 443

Glucoronic acid 221

Glucose

- general 215*, 219*, 221*, 223*, 299*

- output 292*

- space 443

- Tm 386

- tolerance 110

- transport 304*

- utilization 292*

Glucose-6-phosphatase 27, 116, $413 *$

Glucose-6-phosphatase dehydrogenase 5, 410*

Glucose-6-phosphate 116, 202*

Glucosuria 304*

$\beta$-Glucuronidase 306*

Glucuronyl transferase 204*

Gluten enteropathy 27

Glycine 417*

Glycogen 116, 203*220*

Glycogen, liver 443

Glycogenosis $413^{*}, 414^{*}$

Glycogenosis, cardiac 202*

Glycolipidosis $416 *$

Glycolysis 200*, 215*

Goldman, A.S. 217*

Goldman, H. 212*

Goop, R.A. $210^{*}$

GOODNER, C. J. 443

GotLin, R. 203*

Gozzi, E.K. 297*

Graft-vs-host reaction 218*, 317*

GrahAM, G. 219*

Granulocytes 417*

Granulomatosis 209*, 306*, 417*

Gray, J. 311*

GraZianI, L. 225*

GREenBerG, R. E. 292*

Griffin, G. 24

Grose, J. 219*

Growth

- abnormal 205*, 208*

- failure $81,323 *, 417 *$

- general 59, 185, 210*, 266, $417 *$

- organ 185

Growth hormone 202*, 204*, $205 *, 299 *, 300 *, 308 *$

Grumbach, M. M. 308*

Gunn rat $213 *$

GUTHRIE, R.A. 203*

GYLAND, S.P. 169

Hadley, K. 211*

Haessler, H. A. 291*

HAGGE, W. 409*
Hahn, D.A. 169

HALAC, E. 221*

Hamilton, J.R. 220*, 341

HAMMERSTROM, S. 418*

Hammond, J. 294*

Haptoglobin 206*

HARRIS, R. C. 220*

Haslam, R. 219*

Hathaway, W.E. 213*

HAXDEN, P. 224*

Hazeltine, F.G. 223*

Heart 122

Heart block 202*

Heart disease, congenital 201*, 207*

HegaB, H.H. 221*

Heick, H.M. G. 173

Heinz body $216 *$

Hematocrit 169, 288*

Hemoglobin, fetal 410*

Hemoglobinopathy 169

Hemolysis $410^{*}$

Hemolytic uremic syndrome 211*

Hemophilus influenza, infection $216^{*}$

Heparin $211^{*}, 220 *$

Hepatitis, infectious 207*, 294*

Hepatitis, neonatal 409*

HERD, J.K. 203*

Herdman, R. G. 210*

Hereditary disease 14, 386

Herndon, R. 315*

Herpes simplex virus 209*

HeRschkowITZ, N. 223*

HewitT, J. 215*

HEymann, M.A. 287*

Heymann, W. 211*

Highsmith, A.K. 209*

Histamine 216*

HitzIg, W. 417*

HoDGKIN, W.E. 208*

Hodgman, J.E. 303*

Holliday, M.A. 185, 212*

Holtzman, N.A. 219*, 224*

HoNG, R. 217*

Hope, J.W. 314*

HORWITZ, M. 210*

HostetTer, H. G. 213*

Host resistance $319^{*}$

Howell-Jolly body 216 *

HrBeK, A. 416*

HSIA, D.Y.Y. 218*

Hsu, T.H.J. 301*

Hunter-Hurler syndrome 202*

HuRwitz, R. 414*

Hurwitz, R. A. 202*

Hyaluronic acid 203*

Hydrocephalus 201*

Hydrogen ion clearance index 452

Hydrogen ion excretion $212 *$

Hydrops $321^{*}$

$\beta$-Hydroxybutyrate 220 *

5-Hydroxyindoleacetic acid 207*

C-21 Hydroxylase deficiency $415^{*}$

Hydroxyproline 266

Hyperaldosteronism 204*

Hypercapnia 222*, 277, 418*

Hyperglycemia 443
Hyperlipemia 211*

Hyperlipogenesis $291 *$

Hypernatremia 224*

Hyperphagia 291*

Hypersensitivity 208*, 216*

Hypertelorism 206*

Hypertension 204*

Hypertonicity 224*

Hyperuricacidemia 5

Hyperventilation 277, 354

Hypoalbuminemia 211*

Hypocalcemia 219*, 271, 425

Hypocapnia 354

Hypoglycemia 223*, 293*, 299*, $300 *, 413 *$

Hypoglycemia, ketotic $220 *$

Hypogonadism 308*

Hypophosphatasia 314*

Hypophosphatemia 425

Hypophysectomy 204*

Hypopituitarism 299*, 308*

Hypoproteinemia 333

Hypothalamus $202 *, 205 *$

Hypothyroidism 203*, 223*, 271

Hypotonia 206*, 291*

Hypoxemia 200*, 201*, 222*, $410^{*}, 412$

IMMONEN, P. 413*

Immunoglobulins

- general 413*, 418*, 461

- $\gamma$-A $217 *$

- $\gamma$-M 217*, 413*

Immunohistochemistry $210 *$

Immunology 131

Immunoparalysis 178

Indole 372

3-Indoleacetic acid $418 *$

5-Indole-propionic acid 418*

Infection $209^{*}, 210^{*}, 216^{*}, 217^{*}$

Inheritance, sex-linked 5

Inherited disease 220*, 409*, $415^{*}, 417 *$

Insulin 110, 203*, 205*, 220*, $223 *, 299 *$

Insulin, antibodies 110

Insulinase 203*

Intelligence test 59

Interferon $319 *$

Intestine

- general 27, 214*, 220*, 341, 386

- malabsorption 214*

- villus 341

Intracardiac shunting $202 *$

Intrinsic factor 214*, 418*

Iron $214^{*}$

ISBELL, S.V. 220*

IsLes, T.E. 110

Islets of Langerhans 223*

Isohemagglutinin 216*

Isosorbide 224*

Isozymes 165

JAIN, V. 202*

JAMES, V.L., Jr. 297*

JARMAKANI, M.M. 202*

JARRAH, A. 185

Jaundice $217^{*}, 409^{*}$, 410* 
Jejunum $220 *, 412 *, 417 *$

JOB, J.-C. 271

JohN, T.J. 50

JoHNSEN, S. 291*

JoHNSON, L. 219*

Johnson, P. 215*, 321*

Johnston, F.E. 479

Jos, J. 27

Josey, W.E. 209*

Justrce, P. $218 *$

Kadotani, T. 207*

KAHLE, L.L. 208*

Kamitsuka, P. 294*

KandA, I. 202*

KaPLAN, S. L. 308*

KarAKLIS, A. $410^{*}$

Karyotype 208*

KAUDER, E. 208*

KaVeTY, V. 301*

Kekomaki, M. 417*

Kempe, H.C. 210 *

$\alpha$-Ketoglutarate $212 *$

Ketosis 219*, 220*

17-Ketosteroid 203*

Kevy, S.V. 216*

Krazin, A. 290*

KIDD, P. 294*

Kidney

- biopsy $201 *, 211^{*}$

- deoxyribonucleic acid 212*

- function tests 81

- general 39, 91, 99, 185, 206* $207 *, 211 *, 212 *, 219 *, 386$, 452

- hyperplasia $212 *$

- renal biopsy 413*

- transport $212 *$

- tubule 219*

Kinky hair disease 206*

KJELLMER, I. 412*

KLAUS, M. 222*

$\mathrm{KocH}$, A. $417 *$

KoHn, G. 461

Kohrman, A.F. 292*

Kooн, S.W. 425

Korsch, B. M. 297*

Kouvalainen, K. 413*

KrasilnIKOFF, P. 418*

Kretchmer, N. 414*

Krovetz, L.J. 122, 202*

KRUGMAN, S. 217*, 294*

KuITUNen, P. 417*

KuloviaH, M.V. 290*, 237, 247

Kynurenine 372

Lactase 27

Lactate 221*

Lactic acid 215*, 220*, 341

Lactic dehydrogenase 116

LAGERGRANTZ, R. 418*

LAMBERTZ, J. 271

LAMPKIN, B. C. 214*

LAMY, M. 27

Larynx 222*

LAUNIALA, K. $417 *$

Leboeuf, G. 204*

Lecithin 237, 247, 290*

LEES, M.H. 201*
LEIDY, G. $310^{*}$

LEIKIN, S. 214*

LESTRADET, J. 413*

Leucine aminopeptidase 27

L-leucine $220^{*}$

Leukemia 116

Leukocyte 208*, 216*, 315*

Leukocyte NADH oxidase $306 *$

Leukoencephalopathy, subacute sclerosing $315^{*}$

Levine, A. $317 *$

Levine, O.R. 201*

LEWY, J. 212*

LiKLY, B.F. 312*

LINDBERG, O. 411 *

Linoleic acid 364

Lipogenesis 291*

Lipolysis 291 *

Lipoperoxidation 206*

LISCHNER, H.W. 218*, 317*

Liv, H.Y. 386

Liver 116, 165, 178, 206*, 216*, 218*, 220*, 221*, 364, 409*, $414 *$

LOCKWOOD, M.A. 104

LOEB, H. 416*

LoGAN, W.S. 173

LOPATA, E. 200*

LOWE, C.U. 417*

LUCEY, J.F. 215*, 223*

Lunding, M. 418*

Lung

- general 222*, 223*, 237, 247 $290 *, 364,412 *$

- pathology $222 *$

Luteinizing hormone 308*

LYMAN, M. 216*

Lymphocyte 208*, 218*, 418*

Lysine $417^{*}$

Macasaet, F. 294*

MacGillivray, M.H. 205*

Macroglobulin 173

Macrophage 217*, 218*

Magnesium 201*,203*

Malabsorption syndrome 27,412*

MALAN, A.F. 311*

Malate dehydrogenase 205*

Malformation, congenital 206*

Maltase acid 414*

Mangos, J.A. 314*, 436

Mannitol 224*

Marasmus 214*, 219*

Margolis, A. 215*

Martin, J. M. 300*

Marubini, E. $417 *$

Maternal antibody 50

Mathieu, H. 415*

Matsaniotis, N. 418*

Mattioli, L. 222*

Mauer, A.M. 214*, 216*

MAX, C.D. $216^{*}$

MAYall, B.H. 461

McAdams, A. J. 211*

MaCANN,M.L. 312*

MaKAy, R. J., Jr. 208*

MaKhann, G. M. 223*, 315*

MaLoughlin, T.G. 122, 202*

MaSherRy, N.R. 314*, 436
Measles 210*

Megaloblastosis 214*

Mellins, R.B. 201*

Mellman, W.J. 461

MELNICK, J. L. 208*

Mental retardation 372 , 415*

Merredith, T. 59

Mesangium 211*

Metabolism 165, 185, 386

Metaca techoloamine 418*

Metcoff, J. 212*, 413*

Methemoglobinemia 410*

Methylene blue $410^{*}$

MeYer, H. B.P. 222*

Meyer, H. M., Jr. 209*

Michaels, A.J. 372

Microcephaly 207*, 209*,461

Micrognathia 104, 206*

Milhaud, G. 271

Miller, F. 221*

Miller, J.A., Jr. 221*

Miller, M.E. 461

Mitchell, M.B. 122

Mitochondria $200^{*}, 205^{*}, 411^{*}$

Mitosis 208*

Moghadam, A. N. 222*

Money, J. 59

Mongolism 207*

Monkey pox virus 294*

Monoamine oxidase 27

Montgomery, J.R. 208*

Moon, J. B. 277,333

Moore, B. F. 386

Moreno, H. 208*

Morquio's syndrome 418*

MorRelL, F. 225*

Morris, E. 225*

Mosaicism 302*, 461

Motoyama, E.K. 237, 290*

Mucopolysaccharide 203*, 416*, $418^{*}$

Mumps vaccine 209*

Muranyi, L. 411*

Muscular dystrophy $414 *$

Muscular dystrophy, pseudohypertrophic 415*

Myalgia 210*

Mycoplasma pneumoniae 309*

Myelin 223*

Myocardium 202*

$\beta$-Myristic acid 290*

Myxedema 203*

NADER, P.R. $210^{*}$

NAEYE, R. L. 207*

Nahmias, A.J. 209*

NAIB, Z. M. 209*

NaIman, J. L. 309*

NajJAR, V.A. 295*

NATHAN, D.G. 306*

NatzschKa, J.C. 213*

NaUghton, M.A. 224*

Necheles, T.F. 214*

Nelson, N.M. 223*

Nephrectomy 212*

Nephritis, bilirubin 213*

Nephritis, focal 211*

Nephrotic syndrome 208*, 211*, $212^{*}, 413^{*}$ 
Neutrophil 208*

NEw, M.I. 204*

Newborn

- general 50,110, 213* 215* $219 *, 220^{*}, 221, * 223^{*}, 225^{*}$, $288^{*}, 321 *, 410^{*}, 411^{*}, 416^{*}$, 419*

- hemolytic disease

- ABO 301*

- - Rh 223*, 301*, 317*, 321*

Newcastle disease 208*

Nicolopoulos, D. 418*

Niswander, J.D. 206*

Nitrogen balance $417 *$

p-Nitrophenol 221*

Noise, developmental 206*

Noradrenalin 411 *

NORTHWAY, J.D. 211*

Northway, W.H., Jr. 222*

Nourse, C.H. 223*

Novy, M.J. 354

Nucleic acids $414 *$

5-Nucleotidase 27

Nutrition 214*

Nutrition survey 169

NyHaN, W.L. 5

Obesity 291 *

O'Brien, D. 203* 277

OCKerse, A.B. $217 *$

ODELL, G. B. 213*

O'FLYNN, M.E. 218*

Oidiomycin $217^{*}$

Oligomenorrhea 291*

Ourver, T.K., Jr. 223*

Opisthotonos 206*, 207*

ORT, M. 212*

Oski, F.A. 202*, 215*

Ostenogenesis imperfecta $219 *$

Ouabain 24, 436

Oxygenation 354

Oxygen consumption 185

Oxygen demand $411 *$

Oxygen tension $418^{*}$

Oxymetholone therapy $214 *$

PAIne, R.S. 207*

Palmitic acid 290*, 364

Pancreas 436

Pancytopenia 317 *

Panos, T. G. 209*

Parainfluenza type 3 vaccine 50

Parathyroid gland $219 *, 415^{*}, 425$

Parathyroid hormone 219*, 415*, 425

Parer, J.T. 354

PARKKULAINEN, K. 413*

PARKMAN, P.D. 209*

Parmelee, A.H. 209*

Parotid gland 436

PAssarge, E. 206*

Patient care 297*

Patti, A.A. 203*

PAtrick, J.R. 116

Pearson, H.A. 169, 215*

Pendred's syndrome 223*

Penicillamine 224*

Pentamidine isothionate 131

Pentose shunt $417 *$
Perens, R. 200*

Pergament, E. 207*

Perheentupa, J. 417*

Periosteum 479

Peritoneal dialysis $411^{*}$

Perletti, L. 417*

Perlmann, P. 418*

Pesek, J. 5

Peterson, R. E. 204*

pH 333,354

Phagocytosis 208*

Phenobarbital 221*

Phenylalanine 218*, 372

Phenylalanine hydroxylase 218 *

Phenylketonuria 372

Phibbs, R.H. 211*, 215*, 321*

Philippart, M. 416*

Phosphatase, acid 306*

Phosphatase, alkaline 306*

Phosphatidyl dimethylethanolamine 237,247

Phosphatidylethanolamine 247, 290*

Phosphatidyl inositol 237, 247

Phosphaturia 425

Phosphoethanolamine 314*

Phosphofructokinase 202*

Phospholipids 237, 247, 364

Phosphorus 203*,219*

Phosphorylation $411^{*}$

Phototherapy 215*

Phytohemagglutinin 208*, 217*, 317*

Piedrahita, V. 304*

PIEL, C.F. 211 *

Pierson, M. 415*

Pikkarainen, P.H. 165

Piromen 205*

Pituitary 205*, 299*

Placenta

- blood flow 218*

- circulation 287

- general 218*, 317*, 443

- vascular resistance $287 *$

Platelet 218*

Plethysmograph 222*

Plotkin, S.A. 208*

PLuss, H. 417*

Pneumococcal infection 216*

Pneumococcus 178

Pneumocystis carinii pneumonia 131

Polyuria 386

Porter, F.S. 306*

Porter, I.H. 307*

Postural drainage 222*

Potter, D.E. 185, 212*

Prader-Willi syndrome 291*

Prednisone 214*

Prematurity 204*, 210*, 215*

216*, 219*, 222*, 225*, 303*, $411 *, 418 *$

Preschool children 169

Pressure-Volume curve 201*

Priestley, B. L. 223*

Probenicid 212*

Prod'hom, L. 411*

Propylthiouracil 223*

Protein
- clearance 200*

- intolerance $417 *$

- synthesis 203*

Pteroylglutamic acid 214*

Puberty, precocious 59

Pulmonary

- artery $122,222 *$

- hypertension $320 *$

- vascular bed 200*

- vascular disease $320^{*}$

- vasospasm 201*

PunNetT, H.H. 317*

Purpura 214*

Pyrimidines 414*

Pyrogen 205*

Pyrophosphatase 116, 219*

Pyrophosphate 219*

Pyruvate 221 *

Quie, P.G. 217*

Rarha, N.G.R. 165, 293*

RaWLS, W.E. 208*

Rearick, P.D. 224*

Reaven, G. 292*

Redman, W. 310*

REIMER, K. 24

REISER, L. 301*

REMEN, N. 292*

Renal excretion, acid $212 *$

Renal tubular acidosis 298*

Renal tubules $414^{*}, 417 *$

Renin 204*

RENNER, R. 417*

Respirator 222*

Respiratory cilia 173

Respiratory distress syndrome $215^{*}, 221^{*}, 222^{*}, 288^{*}, 290^{*}$, $311 *, 411 *, 412 *, 418 *$

Respiratory epithelium 173

Retardation

- fetal 218*

- mental 207*, 217*

- motor $217 *$

Reticulocytosis 215*

Reticuloendothelial system 178

Rex, J. 27

$\mathrm{Rh}$ factor $215^{*}$

Richardson, F. 415*

Rickets 425

RNA 203*

Robbins, F. C. $309 *$

Robbins, J.B. 131, 215*

RODBRO, P. 418*

RodrigueZ-TORREs, R. 301*

Rogers, L.E. 306*

RondinI, G. 419*

Root, A.W. 202* 314*

Roseman, J. M. 479

Rosen, F.S. $216^{*}$

Rosen, M.G. 226*

RoseNFIELD, R. L. 203*

Rosenkranz, A. 413*

Ross, N.B. 201*

Rossi, E. 413*

Rossier, A. 281

Rousseau, J. 210*

Roy, C. C. $203 *$

ROYER, P. 415* 
Rubella

- congenital 208*

- general 207*,217*

- virus 209*

Rudolph, A. M. 287*

Ruiz, G. 212*

RuttenberG, H.D. 202*

RYLANDER, E. 411 *

SAenz-Rodriguez 299*

SAKAI, T. 212*

Salicylism 277

Saliva 210*, 217*, 314*, 436

Salt-losing syndrome $415 *$

SANDOR, T. 204*

Sarcosine dehydrogenase 409*

Sarcosine hypersarcosinemia 409*

SAuls, H.S. 220*

Schalach, D.S. 308*

Scharer, K. 212*

Schiebler, G. L. 122, 202*

SchindleR, A. M. 207*

SCHNEIDER, J.A. 315*

SCHROTER, W. $410^{*}$

SChUlKInd, M.L. 178

Schulman, I. 39, 99, 196

Schultz, M.A. 225*

SchWARTZ, A. $303 *$

SCRIVER, G. 212*, 219*, 425

SEEGMiller, J.E. $315 *$

SeEler, R. A. 99

Seizure 206*

Self-multilation 5

Septal defect, ventricular $320 *$

Sereni, F. 417*

Serine 247

Serotonin 207*

Sever, J. L. 217*

SEVERI, F. 419*

Shapcotr, D. 203*

SHEIKHOLISLAM, B. M. 205*

Shepard, F. M. 311*

Sherard, E.S. 206*

ShuRTLEFF, D. B. 224*

Shwartzman reaction 39,99

SidBury, J. B. 306*

Steber, O.F., Jr. 50

Sigdell, J. 411 *

SizonenKo, P. 271

Skeletal maturation 470

Skin 210*

SkINNER, R. C. 215*

Sleep 225*

Smith, G. A. 223*

SMITH, C. F. 215*

Smith, C.W. 217*

Smite, F. G., Jr. 212*

SмITH, N.J. 214*

SмITH, R.T. 178

Smits, H.L. 237, 290*

SNIDER, M. 209*

Sodium 436

Sodium, transport 24, 314*

Solomons, C. C. 219*

Soriano, J.R. 81, 298*, 452

Sotos, J.F. 224*

SOURKES, T.L. 293*

South, M.A. 208*

SPACH, M.S. 202*
Sphingomyerin 237, 247

SPLEen 178, 207*, 216*, 218*

SPLENECTOMY $214 *$

Splenomegaly 409*

SPOCK, A. 173

SPRUNT, K. 310*

SRIBNEY, M. 247

Starluman, M.T. $311^{*}$

Staphylococcus aureus 295*

STARK, H. 81, 452

Starling equilibrium $223 *$

Statistics 296*

Steatorrhea 341, 409*

Stekel, A. 214*

Stellate ganglion 202*

Stempfel, R.S. Jr. 205*

STERN, L. 293*

Steroid 211*, 214*

STIEHM, E.R. 221*

Stilbestrol 299*

Storey, G.N.B. 213*

Strabismus 217*

Streptococci, hemolytic 210*, 310*

Strontium, radioactive $414 *$

Stubis, G. 209*

Sugar 220*

Sulfatide $223^{*}$

Sulphate, inorganic $220 *$

Surface-active lipids 364

Surface area 122

Surface tension 237, 247

SUtTERER, W.F. 66

SuvatTe, V. $218 *$

Sweat gland 24

Sweetman, L. 5

SYмснусн, P. 222*

Syphilis $217 *$

Tachycardia, supraventricular $301 *$

Tachycardia, ventricular 202*

Taurine 212*

TAYLOR, P. 200*

Tefft, M. 216*

Teratology $317 *$

Teruya, J. 301*

Testosterone 214*

Tetrahydrocortisol 204*

Tetrahydrocortisone 204*

Tetralogy of Fallot 202*

Tetraploidy 208*, 461

Thalassemia 169, 479

Thermogenesis $411 *$

Thiamine 306*

Thompson, D.J. 443

Thompson, M.M. 300*

Thrombocytopenia 211*, 214*, 216*

Thrupp, L.D. 303*

Thymus $317^{*}$

Thyrocalcitonin 203*, 271

Thyroidectomy 204*

Thyroxine 204*, 223*

Tocopherol 206*

Tooley, W.H. 215*, 321*

Townsend, D. 200*

Toxoplasmosis 217*

Trachea 222*
Transfus on, intrauterine $215 *$, 317*

Translocation 208*, 302*

Transplantation, bone marrow 218*

Travis, L. B. 211*

Triplets 225*

Trishydroxymethylaminomethane (THAM) 221*, 333

Trisomy $302 *$

Tryptophan $372,418 *$

5-oH-Tryptophan 418*

Tsao, M.U. 386

Turner's syndrome 308*

Twins 218*, 410*

Tyrosine $416^{*}$, 418*

UDP-glucuronyltransferase 221 *

UMEZAKI, H. 225*

UMLAUF, H. Y., Jr. 50

5-Bromo-2-Deoxy Uracil 315*

Urea 224*, 417*

Uric acid 5

Uridine diphosphogalactose 4-epimerase 14

Urinary tract infection $413^{*}$

Urine

- amino acid 219*

- concentration 213*

- general 204*, 210*

Umbilical cord 410*

Umbilical cord blood $217 *, 221$ *

UsHeR, R. 221*

Uterine blood flow 354

Vaccinia 210*

VAlAes, T. $410 *$

VANDERHEIDEN, J.F. 39

Vanilmandelic acid 418*

VARGA, F. 410*

Vascular resistance 223*

Vascular responses 223*

Vasoconstriction $412 *$

Vasomotor reflexes $411^{*}$

Vasopressinism 415*

VAWTER, G. 216*

VELASCO, M.S.A. 225*

Ventilation artificial 222*, 311*, 418*

Ventricle

- general 122, 202*

- pressure 224*

Verbal interaction 297*

VERT, P. 415*

VIA, M. 221*

VIDYASAGAR, D. 219*

Viral infection, congenital 217*

Viremia 209*, 294*, 319*

Virus infection, general 319*

VISAKORPI, J. 417*

Viscosity, blood 288*

Vitamin $B_{12} 214 *$

Vitamin C 410*

Vitamin D 219*, 271, 425

Vitamin K 409*

Vomiting 207*

Von Willebrand's disease 213*

Vossough, P. 214* 
WALKER-SMITH, J.A. 412*

Warshaw, J. B. 200*

Water, free clearance $415^{*}$

Waterman, V. 200*

WATSON, D. 200*

WAY, R.C. 201*

WEECH, A. A. 296*

WEIDMAN, W.H. 320*

WEIL, W.B., Jr. $417^{*}, 470$

Werppl, G. 410*

Werss, P. 221*

Weitzman, E. 225*
WENNER, H. A. 294*

WENNER, W. H. 225*

WERTELECKI, W. 207*

West, C.D. $211 *$

Wheal 216*

WHEELER, W.E. 297*

Williams, M.L. 222*

Wrison, I. $411^{*}$

Wilson's disease 219*, 224*

Winick, M. 323*

Wiskott-Aldrich syndrome 216 *

Witte, E.H. 208*
Wood, E. H. 320*

Yaba virus 294*

YAFFE, S.J. 317*

Yolk sac 216

YosHidA, T. 212*

Young, W. C. 311*

YounoszaI, M.K. 266

ZITEL, J. L. 211*

Zuelzer, W.W. 301*

ZuPPINGER, K. 413* 